\title{
In-vitro anti-Vibrio spp. activity and chemical composition of some Tunisian aromatic plants
}

\author{
Mejdi Snoussi · Hafedh Hajlaoui · Emira Noumi · \\ Donatella Usai · Leonardo Antonio Sechi · \\ Stefania Zanetti · Amina Bakhrouf
}

Published online: 18 September 2008

(C) Springer Science+Business Media B.V. 2008

\section{Erratum to: World J Microbiol Biotechnol \\ DOI 10.1007/s11274-008-9811-6}

Due to an unfortunate misunderstanding a typographical error occurred in Table 1 of the above-mentioned publication. The value of Borneol in the fourth plant erroneously reads 60.14 while it should have read: 6.14.

The correct Table 1 is published below and should be treated as definitive by the reader:

The online version of the original article can be found under doi 10.1007/s11274-008-9811-6.

M. Snoussi $(\bowtie) \cdot$ H. Hajlaoui · E. Noumi · A. Bakhrouf Laboratoire d'Analyse, Traitement et Valorisation des Polluants de l'Environnement et des Produits, Department of Microbiology, Faculty of Pharmacy, Rue Avicenne, Monastir 5000, Tunisia

e-mail: snmejdi@yahoo.fr

M. Snoussi - D. Usai · L. A. Sechi · S. Zanetti

Dipartimento di Scienze Biomediche, Sezione di Microbiologia Sperimentale e Clinica, Universita di Sassari, Viale San Pietro 43/b, 07100 Sassari, Italy
Table 1 The main components identified from the five essential oils used in this study

\begin{tabular}{llllll}
\hline Compounds & 1 & 2 & 3 & 4 & 5 \\
\hline$\alpha$-Pinene & $\mathbf{4 . 2}$ & $\operatorname{tr}$ & - & 13.45 & $\mathbf{2 . 8 3}$ \\
Camphene & 0.40 & $\operatorname{tr}$ & - & $\mathbf{5 . 8 6}$ & 0.29 \\
$\beta$-Pinene & 1.06 & $\operatorname{tr}$ & - & 8.36 & 0.23 \\
Myrcene & 0.39 & $\operatorname{tr}$ & - & 1.48 & $\mathbf{2 . 4 8}$ \\
1,8 -Cineole & 10.88 & $\operatorname{tr}$ & - & 43.49 & 0.48 \\
$\gamma$-Terpinene & $\operatorname{tr}$ & $\operatorname{tr}$ & - & 1.0 & 11.20 \\
P-Cymene & $\operatorname{tr}$ & 0.34 & - & 1.39 & 7.58 \\
Menthone & 12.38 & 9.34 & - & - & - \\
Isomenthone & $\mathbf{2 . 5 0}$ & 19.05 & - & - & - \\
Camphrene & - & - & - & 8.95 & $\mathbf{2 . 0 5}$ \\
Bornyl acetate & - & - & - & $\mathbf{4 . 1 3}$ & $\mathbf{4 . 9 3}$ \\
Pulegone & 48.04 & 44.27 & - & - & - \\
Borneol & - & - & - & $\mathbf{6 . 1 4}$ & 0.77 \\
Piperitone & 0.94 & 10.44 & - & - & - \\
Eugenol & - & - & 88.58 & - & - \\
Carvacrol & - & - & - & - & 60.27 \\
Eugenyl acetate & - & - & 5.62 & - & - \\
\hline
\end{tabular}

The numbers 1-5 represent, respectively, the five oils tested in the present study extracted from: Mentha longifolia; M. pulegium; Eugenia caryophyllata; Rosmarinus officinalis and Thymus vulgaris

The values in bold and in italics represent the main components found in the essential oil of tested plants 